\title{
Comparison of Leads Mapping in the Arctic Ocean Between Landsat and MODIS Ice Surface Temperature Products
}

\author{
Xiaoping Pang ${ }^{\mathrm{a}}$, Pei Fan ${ }^{\mathrm{a}}$, Xi Zhao ${ }^{\mathrm{a}}$ *, Qing Ji ${ }^{\mathrm{a}}$ \\ ${ }^{a}$ Chinese Antarctic Center of Surveying and Mapping, Wuhan University, Xiaoping Pang, pxp@whu.edu.cn, Pei Fan, \\ fan_pei@whu.edu.cn,Xi Zhao,xi.zhao@whu.edu.cn, QingJi,jiqing@whu.edu.cn \\ * Corresponding author
}

Keywords: Ice surface temperature, Landsat, MODIS, Leads mapping

\begin{abstract}
:
Leads are linear or wedge-shaped openings in the sea ice cover. They account for about half of the sensible heat transfer from the Arctic Ocean to the atmosphere in winter, though the sea surface area covered by them is only 1\% 2\% of the total sea ice area, thus monitoring leads changes and mapping leads distributions become an essential role on Arctic researches. Sea ice surface temperature (IST) product from Moderate Resolution Imaging Spectroradiometer (MODIS) is the most used source of leads monitoring and mapping, however, due to the coarse spatial resolution (1 km at swath level), it suffers from mixed pixel effect when describes the temperature variations on thin leads (10m 100m), thus an IST product with a finer spatial resolution is needed. Though several surface temperature retrieval algorithms had been introduced based on Landsat 8 thermal imagery, none of them were validated in Arctic sea ice region. Given that the special weather conditions such as air temperature inversion were not taken into consideration, these algorithms may not always suitable for IST acquisition in Arctic. In this paper, we applied five mainstream IST algorithms (three split window algorithms and two single channel methods) on Arctic sea ice, compared the Landsat 8 IST with corresponding MODIS IST product, and validated all the satellite ISTs by in situ temperature measurements from drifting buoys. Compared to the buoy ISTs, the single channel method through web-based atmosphere correction tool provided by Barsi et al. (2003) offers the best accuracy. The split window algorithm proposed by Du et al. (2015) ranks the second, but constrained by the banding effect due to the stripe noise. Split window algorithm introduced by Jiménez-Muñoz et al. (2014) coincides with MODIS IST product best. All of the three methods mentioned above have slightly better accuracy than MODIS IST, particular in thin leads areas, which indicated that Landsat based leads map will provide us a better insight of Arctic sea ice. All the satellite ISTs tend to underestimate the surface temperature than those measured by buoys.
\end{abstract}

